\title{
USING A DENTAL OPERATING MICROSCOPE IN THE TREATMENT OF REVERSIBLE PULPITIS IN PRIMARY TEETH
}

\section{ABSTRACT}

Objectives: The author reports on a study of the application of the magnifying method with a digital operating microscope (DOM) in combination with controlled excavation using fluorescent method (Proface) in the treatment of asymptomatic closed pulpitis in primary molars treated with the indirect pulp capping technique.

Materials and methods: Subject of the study were 40 primary molars (19 first and 21 second molars) of children (20 girls and 20 boys) 4-7 years old with cavitated carious lesions in the dentin suspected for asymptomatic closed pulpitis. All cases were excavated with an identical clinical protocol (conventional excavation). The excavation was controlled using the fluorescent method (Proface). An assessment of the residual dentin, with and without microscope (DOM), at three levels of magnification, was made at each stage.

Results: The use of DOM shows a: larger diversity in the colors and nuances of the carious dentin, with lighter shades being predominant. DOM gives an opportunity for a better precision in determining the speed of the carious process, which is in direct relation to the defensive ability of the pulp-dentin complex.

Conclusions: In the biological treatment of asymptomatic closed pulpitis in primary teeth, the use of DOM magnifying technology gives the opportunity for a precise and accurate assessment during the course of excavation

Keywords: Dental operating microscope, FACE, reversible pulpitis.
Maya RASHKOVA ${ }^{1}$

*Nadezhda MITOVA ${ }^{1}$

Zornitsa LAZAROVA ${ }^{1}$

Natalia GATEVA ${ }^{1}$

ORCID IDs of the authors: M.R. 0000-0001-8236-1085

N.M. 0000-0003-4716-7724

Z.L. $0000-0003-0116-259 \mathrm{X}$

N.G. 0000-0002-2584-7983

${ }^{1}$ Department of Pediatric Dentistry, Faculty of Dental Medicine, Medical UniversitySofia.

Received : :07.11.2019 Accepted : $: 06.02 .2020$ 


\section{INTRODUCTION}

The microinvasive approach in the treatment of caries and its complications is a new paradigm in dental medicine. If the inflammatory changes to the pulp are still reversible (in asymptomatic pulpitis), this approach gives the opportunity to preserve the integrity of the pulp and beneficially influence the healing process. ${ }^{1-5}$ Clinically, an asymptomatic closed pulpitis is accompanied by deep carious lesions in the dentin and its treatment requires a non-invasive controlled excavation. The aim of this is to preserve the reversibly damaged dentin capable of internal remineralization and to also arrest the carious process, to stimulate tertiary dentinogenesis and the healing process in the dental pulp. ${ }^{2-4}$ A precise controlled excavation can be achieved through the use of the appropriate magnifying tools, among which is the dental operating microscope (DOM) combined with caries-detector using laser fluorescence for precise assessment of the degree of demineralization and microbial invasion of the dentin. ${ }^{6-8}$

Three strategies can be found in scientific literature in regard to the microinvasive approach in controlled excavation with both deep caries and treatment of reversible pulpitis. The first is nonselective excavation down to hard dentin, i.e. removal of all the soft dentin until healthy, hard, non-carious dentin is reached. ${ }^{10-12}$ In primary teeth the non-selective excavation of carious dentin down to hard dentin carries a big risk of exposing the pulp and it stands in contradiction to the microinvasive approach which is applied for the treatment of reversible pulpitis. The second strategy is selective excavation down to affected dentin. This is applied in cases of deep dentin caries when the caries has reached the inner third of the dentin. ${ }^{3-5,9,13}$ The third is selective removal of partially infected dentin. This method of excavation is recommended for teeth with asymptomatic closed pulpitis where the inflammation is still reversible. In this case the intention is to avoid unwanted exposure of the pulp and endodontic treatment which is risky for primary teeth with advanced root resorption. ${ }^{11-16}$ Comparative studies have been carried out over the last years on the precision, sensitivity and specificity of various methods for assessing the residual dentin during cavity preparation. Its features play a crucial role in the healing process. The results favor the contemporary fluorescent methods which best objectivize the infected dentin. ${ }^{17-20}$ Light-induced fluorescence has gradually turned into a concept for control of the excavation, thus the problem with the subjective assessment of the operator has been overcome. Combining FACE (Fluorescence-Aided Caries Excavation) technology with digital magnifying technology is a new approach which improves precision in selective excavation of reversible pulpitis in children. ${ }^{19,20}$

In this study, the authors reports of the application of the magnifying method by use of a digital operating microscope (DOM) in combination with controlled excavation with fluorescent method (Proface) in the treatment of asymptomatic closed pulpitis in primary molars treated through an indirect pulp capping.

\section{MATERIALS AND METHODS}

This study was conducted from February 2019 to August 2019 at the Faculty of Dental Medicine, Medical University of Sofia. The children were included in the study after explaining its purpose to their parents/guardians and obtaining their written consent. An ethical approval from KENIMUS - Ethical commission of Medical University of Sofia - 24/07.12.2018 was obtained. The study protocol and design was approved by the KENIMUS.

Subject of the study were 40 primary molars (19 first and 21 second molars) of children (20 girls and 20 boys) 4-7 years old, with deep cavitated carious lesions in the dentin, diagnosed as asymptomatic closed pulpitis. The primary molars with diagnosys asymptomatic pulptis (lack of spontaneous and night pain), included in the study, were selected randomly according to CONSORT guideline and according to the following criteria:

- in approximal lesions - over $2 / 3$ of the approximal surface is affected, presence of a cusp destroyed by caries. 
- in occlusal lesions - the lines of the lesion reach the middle of the distance between the deepest point of the fissure and the top of the closest cusp, no reddening or pain during gingival palpation, no pulp communication, no pain during percussion.

For the purposes of this study a comparative diagnostic with and without DOM (8x, 16x, 23x) was carried out. All cases of asymptomatic closed pulpitis were excavated with an identical clinical protocol (conventional excavation). An assessment of the residual dentin, with and without microscope, was made at each stage. Each stage of the excavation was described in a purpose-made medical card.

The excavation was controlled with the fluorescent method (Proface). In this method of control the tooth is illuminated with violet light up to $405 \mathrm{~nm}$. Glasses with a filter with permeability of up to $500 \mathrm{~nm}$ of the optical spectrum are used. The infected dentin is seen in red, while healthy structures have a greenish color. We applied criteria for fluorescent control with (Proface) for dentin at different stages of destruction: ${ }^{21}$
- infected dentin: the fluorescence is with an intensive red or dark red color and completely encompasses the carious dentin;

- partially infected dentin: pink fluorescence with the presence of some localized limited red spots in the area of the dentin above the pulp;

- affected dentin: pale pink fluorescence localized only in various spots at the bottom of the cavity (the area of the dentin above the pulp), with no fluorescence present in the rest of the cavity;

- healthy dentin: no red fluorescence localized in the dentin-enamel junction and 1-2 $\mathrm{mm}$ from the walls of the cavity.

At each stage of excavation, after reaching the respective fluorescent criterion, the visualtactile characteristics of the residual dentin were registered, both the ones visible to the naked eye and those with DOM, at three levels of magnification. The visual-tactile method of Bjørndal et al. ${ }^{22}$ was used (Table 1).

Table 1. Visual-tactile method of Bjørndal et al. ${ }^{22}$

\begin{tabular}{l|l}
\hline Visual criteria - dentin color & Tactile criteria (with probe) -dentin consistency \\
\hline $\begin{array}{l}\text { black } \\
\text { dark brown }\end{array}$ & $\begin{array}{l}\text { Code 1 (very soft) - the probe penetrates and peels parts of it } \\
\text { away easily; } \\
\text { Code 2 (soft dentin) - probe can easily penetrate and exit the } \\
\text { dentin; } \\
\text { light brown }\end{array}$ \\
$\begin{array}{l}\text { Code 3 (moderately hard) - slight resistance during probing; } \\
\text { Code 4 (hard dentin) - the probe moves over the dentin with a } \\
\text { slight resistance, and leavs a white trail; } \\
\text { Code 5 (hard non-carious) - soft squeaking and resistance during } \\
\text { probing. }\end{array}$ \\
\hline
\end{tabular}

\section{Clinical Protocol}

- Stage 1: exposing the carious lesion and excavation down to healthy dentin along the dentin-enamel junction (DEJ) and gingival base;

- control with Proface down to no presence of red fluorescence;

- description of visual-tactile characteristics of dentin (visible to the naked eye, with DOM with three levels of magnification (8x, 16x, 23.3x); photo documentation;
- Stage 2: excavation of carious dentin down to fluorescent criteria for partially infected dentin with Proface;

- control with Proface - pale pink fluorescence of the dentin above the pulp with limited spots of red fluorescence in the deepest areas;

- description of visual-tactile characteristics of the partially infected dentin (visible to the 
naked eye, with DOM with the same three levels of magnification; photo documentation;

- calcium hydroxide cement application (caviLINE - AHL) and temporary filling with glass ionomer cement (GIC) (GC FUJI TRIAGE White) for three months;

- X-ray control (bitewing) allowing reproducibility of the image at the subsequent control radiographic examination in childhood.

- Stage 3: monitoring

- after one month: clinical monitoring and evaluation of the integrity of the GIC filling and the symptoms present;

- in the third month: X-ray control - assigned radiography with the same exposure parameters as the diagnostic one;

- revision of the cavity: if necessary, an excavation is carried out (if there are fluorescent

Table 2. Consistency of the carious dentin during lesion exposure

\begin{tabular}{|c|c|c|c|c|c|c|c|}
\hline \multirow{2}{*}{$\begin{array}{l}\text { Consistency } \\
\text { localization }\end{array}$} & \multicolumn{3}{|c|}{ Very soft } & \multicolumn{2}{|l|}{ soft } & \multicolumn{2}{|c|}{ tal } \\
\hline & $\mathrm{N}$ & $\% \pm s p$ & $\mathrm{~N}$ & $\% \pm \mathrm{sp}$ & $\mathrm{N}$ & $100 \%$ & \\
\hline occlusal & 13 & $81.3 \pm 9.76$ & 3 & $18.7 \pm 9.76$ & 16 & $100 \%$ & $\begin{array}{l}\mathrm{T}=4.53 \\
\mathrm{p}<0.05\end{array}$ \\
\hline approximal & 19 & $79.2 \pm 8.29$ & 5 & $20.8 \pm 8.29$ & 24 & $100 \%$ & $\begin{array}{l}\mathrm{T}=4.98 \\
\mathrm{p}<0.05\end{array}$ \\
\hline & $\mathrm{T}=0.16$ & .05 & $\mathrm{~T}=0$ & .05 & & & \\
\hline
\end{tabular}

Pearson Chi Square $=0.417 \quad$ Sig $=0.519$

The consistency of the carious dentin during lesion exposure is predominantly very soft regardless of the localization of the lesions. The differences are supported by statistical plausibility $(\mathrm{p}<0.5)$.

Table 3. Color of the dentin during carious lesion exposure without and with the use of a DOM ${ }^{\mathrm{i}}$ with $8 \mathrm{x}, 16 \mathrm{x}$ and $23.3 \mathrm{x}$ magnification

\begin{tabular}{lcccccccc}
\hline \multicolumn{1}{c}{ Color } & \multicolumn{2}{l}{ Dark brown } & \multicolumn{2}{c}{ Light brown } & \multicolumn{2}{c}{ Dark yellow } & \multicolumn{1}{c}{ total } \\
\cline { 2 - 9 } & $\mathrm{N}$ & $\% \pm \mathrm{sp}$ & $\mathrm{N}$ & $\% \pm \mathrm{sp}$ & $\mathrm{N}$ & $\% \pm \mathrm{sp}$ & $\mathrm{N}$ & $100 \%$ \\
\hline No microscope & 16 & $40.0 \pm 7.75$ & 24 & $60.0 \pm 7.75$ & 0 & $0 \pm 0$ & 40 & $100 \%$ \\
With micro. - 8x & 14 & $35.0 \pm 7.54$ & 16 & $40.0 \pm 7.75$ & 10 & $25.0 \pm 6.85$ & 40 & $100 \%$ \\
With micro. - 16x & 14 & $35.0 \pm 7.54$ & 16 & $40.0 \pm 7.75$ & 10 & $25.0 \pm 6.85$ & 40 & $100 \%$ \\
With micro. - 23.3x & 14 & $35.0 \pm 7.54$ & 16 & $40.0 \pm 7.75$ & 10 & $25.0 \pm 6.85$ & 40 & $100 \%$ \\
& $\mathrm{~T}=0.46$ & $\mathrm{p}>0.05$ & \multicolumn{2}{c}{$\mathrm{T}=1.83 \mathrm{p}>0.05$} & & & & \\
\hline
\end{tabular}

Pearson Chi Square $=12,470 \quad$ Sig $=0,002$
The data shows, that when a DOM with $8 x$ magnification is used, a larger diversity of colors red areas at the bottom of the cavity), calcium hydroxide liner (caviLINE - AHL) is re-applied, and a definitive filling with a compomer is carried out (Dyract ${ }^{\circledR}$ XP - Dentsplay Siron).

Later results will be subject to a subsequent publication.

For the statistical processing of the results a statistical program SPSS (version 19, SPSS inc. USA) was used. A 95 confidence interval $(\mathrm{p}<0.05)$ was chosen for a level of plausibility at which the null hypothesis is to be rejected.

\section{RESULTS}

1. Description of the carious dentin after The results from the visual-tactile description of the carious dentin with and without DOM are presented Table 2.
Table 3 presents the results of the determination of the color characteristics of the dentin upon exposing of the carious lesion. 
visual assessment with no magnification apparatus

(fig.1).

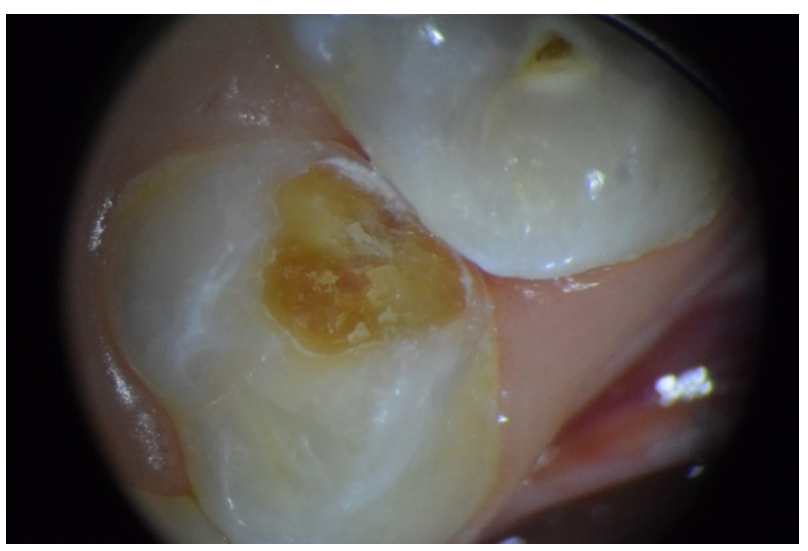

Figure 1. Photo of the approximal lesion of the left maxillary first primary molar (DOM 8x). Carious dentin with a soft and moist texture is observed during lesion exposure.

No plausible differences in the registered color of the dentin between the various degrees of magnification are observed when using a DOM ( $>0.05)$.

\section{Description of the carious dentin in the} DEJ area after excavation with and without DOM

In the DEJ area, after controlled excavation down to non-carious, healthy dentin (lack of fluorescence with Proface), the consistency of the dentin is code 5 by Bjørndal et al. ${ }^{22}$ - hard dentin, matching the description of non-carious, observed in all clinical cases.

In all clinical cases, when excavating down to healthy dentin and when assessing with and without DOM, a light-yellow dentin is observed matching the characteristics of a healthy structure, with no difference in the assessment being observed with and without DOM.

\section{Description of the residual carious dentin} in the area at the bottom of the cavity observed with and without DOM

After excavationof the DEJ, carious dentin remains at the bottom of the lesion which with Proface fluoresces in red.

The consistency of the residual carious dentin is predominantly soft regardless of the localization of the lesion (approximal or occlusal).

The comparative results regarding the color of the infected dentin are presented in Table 4 .

Table 4. Color of the infected dentin at the bottom of the lesion without and with the use of a microscope with $8 x$, $16 x$ and 23.3x magnification

\begin{tabular}{|c|c|c|c|c|c|c|c|c|}
\hline \multirow{2}{*}{ Magnification } & \multicolumn{2}{|c|}{ Dark brown } & \multicolumn{2}{|c|}{ Light brown } & \multicolumn{2}{|c|}{ Dark yellow } & \multicolumn{2}{|c|}{ total } \\
\hline & $\mathrm{N}$ & $\% \pm \mathrm{sp}$ & $\mathrm{N}$ & $\% \pm \mathrm{sp}$ & $\mathrm{N}$ & $\% \pm \mathrm{sp}$ & $\mathrm{N}$ & $100 \%$ \\
\hline No microscope & 16 & $40.0 \pm 7.75$ & 24 & $60.0 \pm 7.75$ & 0 & $0 \pm 0$ & 40 & $100 \%$ \\
\hline $\begin{array}{l}\text { With microscope } \\
-8 x \text { magnification }\end{array}$ & 7 & $17.5 \pm 6.01$ & 21 & $52.5 \pm 7.90$ & 12 & $30.0 \pm 7.25$ & 40 & $100 \%$ \\
\hline $\begin{array}{l}\text { With microscope }-16 x \\
\text { magnification }\end{array}$ & 7 & $17.5 \pm 6.01$ & 21 & $52.5 \pm 7.90$ & 12 & $30.0 \pm 7.25$ & 40 & $100 \%$ \\
\hline \multirow[t]{2}{*}{$\begin{array}{l}\text { With microscope - } \\
23 x\end{array}$} & 7 & $17.5 \pm 6.01$ & 21 & $52.5 \pm 7.90$ & 12 & $30.0 \pm 7.25$ & 40 & $100 \%$ \\
\hline & \multicolumn{2}{|c|}{$\mathrm{T}=2.30 \quad \mathrm{p}<0.05$} & \multicolumn{2}{|c|}{$\mathrm{T}=0.68$} & \multicolumn{2}{|c|}{$\mathrm{T}=4.140 \mathrm{p}<0.05$} & & \\
\hline
\end{tabular}

Pearson Chi Square $=13.611 \quad$ Sig $=0.001$

The color of the carious dentin at the bottom of the lesion, observed under a DOM with $8 \mathrm{x}$ magnification displays statistical plausibility, greater diversity of colors, and a stronger predominance of the lighter shades in comparison with the color of the same dentin when observed without a microscope $(p<0.05$, Table 4$)$. No difference can be seen in the various levels of magnification.

Here is a comparison of the carious dentin observed with DOM on the surface of the lesion before excavation, and the one at the bottom of the cavity after DEJ excavation. The results are presented in the Figure 1. 


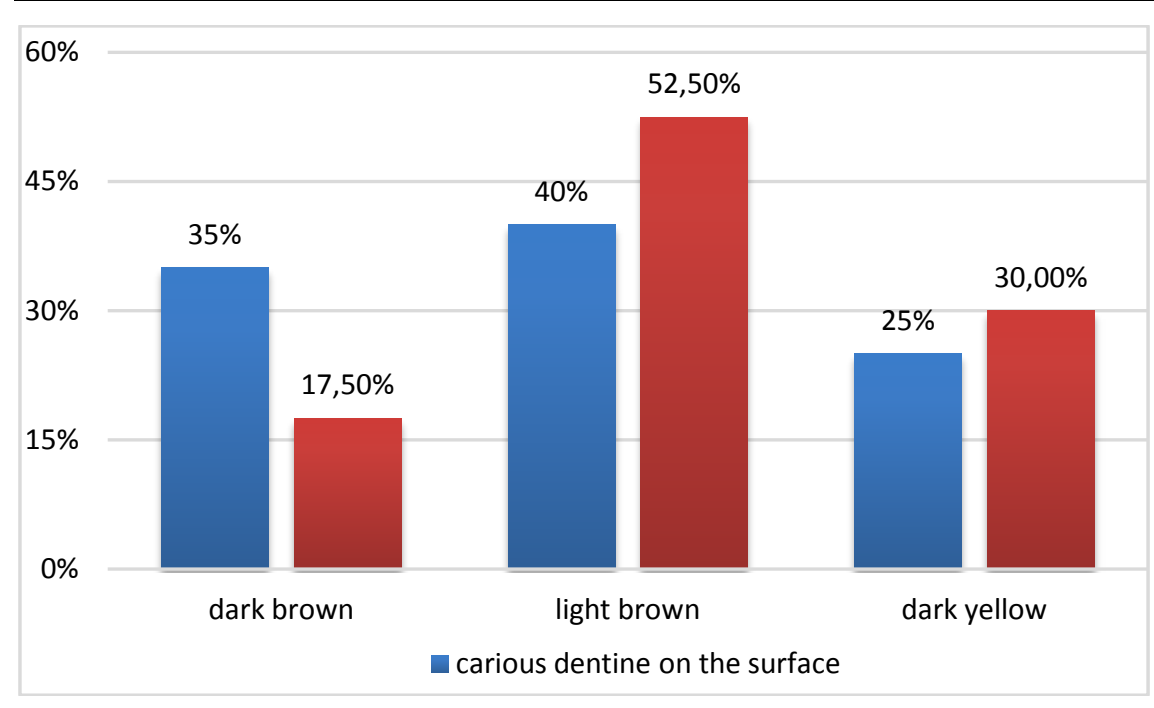

Figure 1. Comparison of the color of the surface carious dentin (before excavation) with the one at the bottom of the lesion, with DOM ${ }^{\mathrm{ii}} 8 \mathrm{x}$

Predominant on the surface is the dark carious dentin. At the bottom there is a greater diversity, with dark and light brown dentin being evenly distributed in about half of the lesions, while lighter shades are observed in a third to a quarter of the cases.

\section{Description of the partially infected circumpulpal dentin, capable of internal re- mineralization - observed with and without \\ DOM}

In clinical cases of asymptomatic closed pulpitis, in the partially infected dentin, which can be preserved, has been shown to be able to stimulate tertiary dentinogenesis and a healing process in the area of the underlying pulp horn, affected by the initial pulp inflammation. The results regarding the color of the partially infected circumpulpal dentin are presented in the Table 5.

Cases with a dark yellow circumpulpal dentin color are predominant, both with DOM and to the naked eye $(p<0.05)$. In two thirds of the cases the color of the dentin studied is yellowish and in the rest of the cases it is light brown, and in only three of the cases it is with darker shades, the ratio between the colors being preserved with and without DOM ( $>0.05$, Table 5).

Table 5. Color of the partially infected circumpulpal dentin without and with the use of a $\mathrm{DOM}^{1}$ with $8 \mathrm{x}, 16 \mathrm{x}$ and $23.3 \mathrm{x}$ magnification

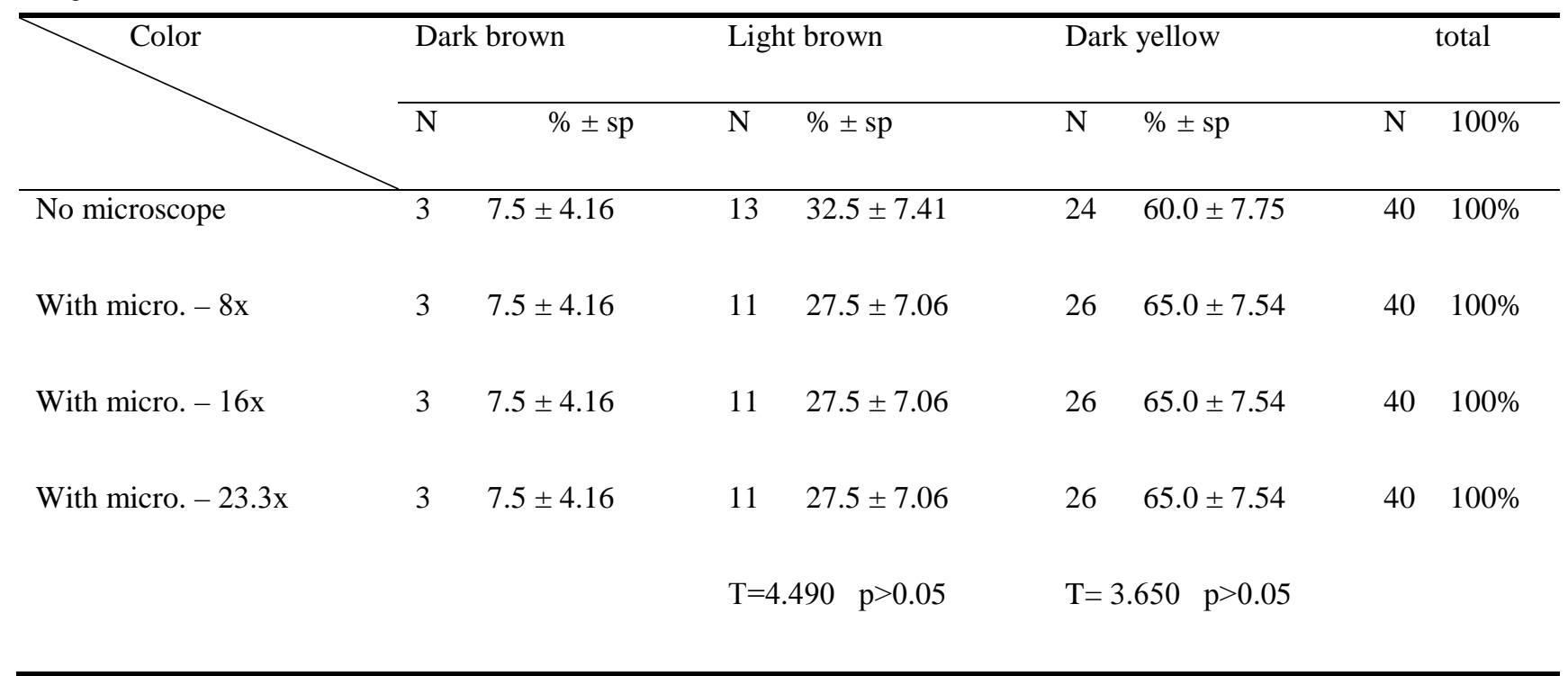


The consistency of the partially infected dentin was assessed as averagely hard in all clinical cases studied - with code 3 (moderately hard dentin offering light resistance during probing under the Bjørndal et al. ${ }^{22}$ ) (Fig.2).

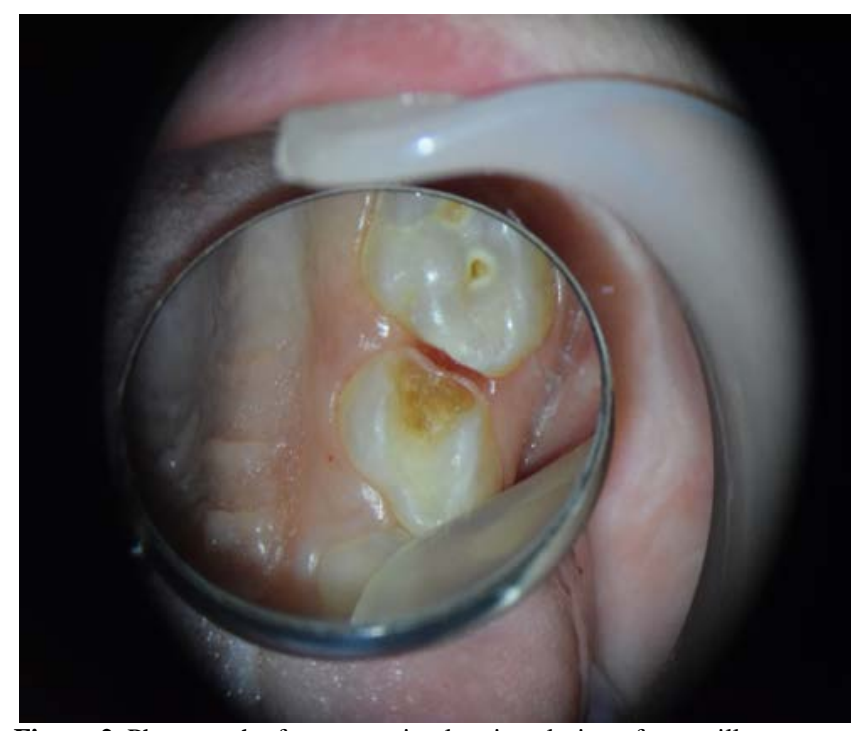

Figure 2. Photograph of an approximal carious lesion of a maxillary first left primary molar made with DOM at magnification $8 x$. Presence of partially infected dentine at the bottom of the cavity that may be preserved.

The use of DOM enables a more precise assessment of the partially infected dentin, with the aim being to preserve the dentin and arrest the carious process.

\section{DISCUSSION}

In our study we found that when using a DOM with magnification $8 x$, a larger diversity of colors can be seen, with lighter shades of carious dentin being predominant, in comparison with the clinical-visual assessment with no magnification apparatus.Lighter shades of carious dentin are an indicator of faster developing caries lesions, with faster advancement towards the pulp and a weaker immune reaction on part of the odontoblasts. In such cases, slowing down the process is one of the aims of the treatment. It is achieved through limiting the microbial invasion. In our study we found, that no plausible differences in the registered color of the dentin between the various degrees of magnification were observed when using a DOM ( $\mathrm{p}>0.05)$.

We found that the color of the carious dentin at the bottom of the lesion, observed under a DOM with magnification $8 x$, displays statistical plausibility, greater diversity of colors, and a stronger predominance of the lighter shades in comparison with the color of the same dentin, when observed without a microscope $(\mathrm{p}<0.05$, Table 4). No difference can be seen in the various levels of magnification. The color of the carious dentin is related to the speed of development and progression of the carious process, which depends on the effectiveness of the defense reaction of the pulp-dentin complex, as well as on the severity of the inflammatory reaction in the pulp, and, to a degree, the reversibility of the inflammatory process. ${ }^{9-12,14,23}$ The possibility of a more accurate assessment of the color characteristics of carious dentin when using DOM, regardless of the degree of magnification, provides an opportunity for a more accurate prognostic of the healing process.

In our study the dark carious dentin is predominant on the surface dentin. At the bottom, there is a greater diversity, with dark and light brown dentin being evenly distributed in about half of the lesions, while lighter shades were observed in a third to a quarter of the cases. Using DOM provides a greater accuracy and precision in the clinical visual assessment of the dentin present and it allows us to register some visible lightening of the carious dentin in its deep layers, which still corresponds to red fluorescence under the cariesdetector. This fluorescence indicates an irreversible destruction and a strong microbial invasion, as well as faster advancement of the process towards the pulp. ${ }^{19,21}$

In clinical cases of asymptomatic closed pulpitis, the partially infected dentin which can be preserved, has been shown to be able to stimulate tertiary dentinogenesis and a healing process in the area of the underlying pulp horn, affected by the initial pulp inflammation. This dentin meets the Proface criterion: pink fluorescence with the presence of localized limited red spots in the area above the pulp. This fluorescence is an indicator of irreversible destruction of the dentin and strong microbial invasion, which favors a faster progressing of the inflammation towards the pulp. ${ }^{9,11,14}$ In our study the results regarding the color of the partially infected circumpulpal dentin 
are presented in the Table 5. Predominant are the cases with a dark yellow circumpulpal dentin color, visible both with DOM and to the naked eye $(\mathrm{p}<0.05)$.

A correct and precise assessment of this dentin is particularly important, considering the small volumes of dental structures found in primary teeth. The use of magnifying technology is an important condition for the correct treatment, the preservation of integrity of the pulp and for applying the biological approach.The use of DOM enables a more precise assessment of the partially infected dentin, with the aim being to preserve the dentin and arrest the carious process. The use of magnifying technology is an excellent diagnostic method for applying the microinvasive approach in the biological treatment of reversible pulpitis. DOM-assisted assessment gives more opportunities for preserving the partially infected dentin, which ensures the integrity of the pulp chamber and thus we try to stimulate internal remineralization, tertiary dentinogenesis, as well as the healing process in the pulp. Specialized literature offers no researches on the use of DOM as an assistance to selective excavation in the treatment of reversible pulpitis, although there are various authors reporting on their studies aimed at seeking and developing a number of methods for biological treatment of reversible pulpitis. ${ }^{12-14}$ This study shows, that the use of DOM is an asset to controlled excavation with a view to the clinical assessment of the changes of the color of the dentin, and it provides a more accurate overall assessment compared to conventional visualtactile diagnosis (Tables 3,4,5). Assessment of changes in the color characteristics of the dentin is an important diagnostic indicator for a noninvasive and precise approach in the biological treatment of pulpitis in primary teeth. ${ }^{11,14}$ Differentiating the infected dentin at various degrees of demineralization is particularly important in reversible pulpitis, mostly with a view to applying indirect pulp cupping as a method of treatment. ${ }^{11,13,14,23}$

\section{CONCLUSIONS}

- In the biological treatment of asymptomatic closed pulpitis in primary teeth, the use of DOM magnifying technology gives the opportunity for a precise and accurate assessment during the course of excavation.

- The use of DOM shows a: larger diversity in the colors and nuances of the carious dentin, with lighter shades being predominant. DOM gives an opportunity for a better precision in determining the speed of the carious process, which is in direct relation to the defensive ability of the pulp-dentin complex;

- The use of fluorescent control with Proface is recommended during the course of excavation, and the use of magnifying technology complements the objective assessment in the area of the circumpulpal dentin which, in reversible pulpitis of primary teeth, must be preserved and used for the stimulation of the healing process.

\section{ACKNOWLEDGEMENT}

This work was supported by the Council of Medical Science at Medical University of Sofia, Bulgaria under Infrastructure Project with Contract No. 207 / 12.12.2018.

\section{CONFLICT OF INTEREST STATEMENT}

The authors declare that they have no competing interests.

\section{REFERENCES}

1. Peneva M, Kabakchieva R, Rashkova M, Gateva N, Doychinova L, Zhegova G, editors. Clinic of pediatric dentistry. $1^{\text {st }}$ ed. Sofia: Bedemot; 2018.

2. Mitova N, Rashkova M, Uzunov T, Kosturkov D, Petrunov V. Controlled excavation for cavitated dentinal caries with visual-tactile method and fluorescence with Proface W\&H. Problems of dental medicine 2014; 40: 13-21.

3. Mitova N, Rashkova M, Zhegova G, Uzunov T, Kosturkov D, Ishkitiev N. Comparison of different methods of excavation control for minimally invasive caries treatment. STOMAEDUJ 2016; 3: 98-106.

4. Mitova N. Minimally invasive approach to dentine caries of permanent teeth in children. PhD Thesis. Sofia; 2016. [In Bulgarian].

5. Mitova N, Rashkova M, Gergova R, Jegova G, Nocheva Hr, Krastev D. Treatment of deep dentinal caries with micro-invasive technique - clinical and 
microbiological evaluation. SYLWAN 2015; 159: 498511.

6. Schwendicke F, Jäger AM, Paris S, Hsu LY, Tu YK. Treating pit-and-fissure caries: a systematic review and network meta-analysis. J Dent Res 2015; 94: 522-523.

7. Murgel C. Microdentistry: Concepts, Methods, And Clinical Incorporation. Int J MicroDent 2010; 2: 56-63.

8. Fanibunda U, Meshram G, Warhadpande M. Evolutionary Perspectives On The Dental Operating Microscope: A Macro Revolution At The Micro Level. Int J MicroDent 2010; 2: 15-19.

9. Ricketts D, Lamont T, Innes NP, Kidd E, Clarkson JE. Operative caries management in adults and children. Cochrane Database Syst Rev 2013; 28:CD003808.

10. Schwendicke F, Paris S, Tu Y. Effects of using different criteria and methods for caries removal: a systemic review and network meta-analysis. J Dent. 2015; 43: 1-15.

11. Schwendicke $\mathrm{F}$ et al. Managing caries removal: consensus recommendations on carious tissue removal. Adv Dent Res 2016; 28: 58-67.

12. Schwendicke F, Frenken J, Innes N. Caries Excavation Evolution of Treating Cavitated Carious Lesions. Removing Carious Tissue: Why and How? Adv Dent Res 2016; 28: 56-65.

13. Innes NP et al. Managing caries lesion: consensus recommendations on terminology. Adv Dent Res 2016; 28: 49-57.

14. Smaïl-Faugeron V, Glenny AM, Courson F, Durieux P, Muller-Bolla M, Fron Chabouis H. Pulp treatment for extensive decay in primary teeth. Cochrane Database of Systematic Reviews 2018; DOI: 10.1002/14651858.CD003220.pub3.

15. Arslan I, Aydınoglu S, Baygin O, Tuzuner T, Sirin M. Comparative Analysis of Manual, Rotary and Reciprocal Systems on Primary Teeth Root Canals: An In Vitro Scanning Electron Microscopy Study. Cumhuriyet Dent J 2019; 22: 299-309.

16. Ziya M, Yüksel BN, Sarı Ş. Root Canal Morphology of Mandibular Primary Molars: A MicroCT Study. Cumhuriyet Dent J 2019; 22:24.
17. Santamaria RM, Innes NP, Machiulskiene V, Evans DJ, Splieth CH. Caries management strategies for primary molars: 1-yr randomized control trial results. J Dent Res 2014; 93: 1062-1069.

18. Iwami Y, Shimizu A, Narimatsu M, Hayashi M, Takeshige F, Ebisu S. Relationship between bacterial infection and evaluation using a laser fluorescence device, DIAGNOdent. Eur J Oral Sci 2004; 112: 419423.

19. Lai G1, Zhu L, Xu X, Kunzelmann KH. An in vitro comparison of fluorescence-aided caries excavation and conventional excavation by microhardness testing. Clin Oral Investig 2014; 18: 599-605.

20.Lennon A et al. Fluorescence-aided caries excavation (face) caries detector and conventional caries excavation in primary teeth. Pediatric Dentistry. 2009; 31: 316-319.

21. Mitova N, Rashkova M. Clinical protocol for microinvasive treatment of deep dentine caries of permanent teeth in children. Problems of dental medicine. 2017; 43: 35-42.

22. Bjørndal L, Larsen T, Thylstrup A. A clinical and microbiological study of deep carious lesions during stepwise excavation using long treatment intervals. Caries Res 1997; 31: 411-417.

23. Schwendicke F, Dorfer CE, Paris S. Incomplete caries removal: a systemic review and meta-analysis. J Dent Res 2013; 92: 306-314. 